\title{
ANÁLISE HORIZONTAL DE LIVROS DIDÁTICOS DE BIOLOGIA DO ENSINO MÉDIO REFERENTE AO CONTEÚDO DE ZOOLOGIA - CLASSE MAMMALIA
}

\author{
Apresentação: Comunicação Oral
}

José Djalma de Souza ${ }^{1}$; Priscila Gomes Coutinho²; Paulo Diêgo Elias do Nascimento ${ }^{3}$; Juliana Rosa Farias da Silva ${ }^{4}$; Argus Vasconcelos de Almeida ${ }^{5}$

\begin{abstract}
Resumo
O livro didático (LD) é um instrumento importante para o ensino formal, mesmo não sendo o único material a ser utilizado em sala de aula, é decisivo para a qualidade do ensino. Uma das condições para que o LD seja bem avaliado é a que esteja com os conceitos cientificamente corretos, com níveis de rigor e precisão apropriados à série que se destina; e possuir narrativas claras e compreensíveis, colocadas em uma linguagem interessante, que estimule o pensamento do educando. Por esse motivo, o presente trabalho objetivou-se em analisar horizontalmente livros didáticos do ensino de biologia usados em escolas do estado de Pernambuco, considerando seu conteúdo teórico, recursos visuais e adicionais e atividades propostas, utilizadas para o ensino da Classe Mammalia. Numa amostra total de seis títulos analisados, a partir dos livros selecionados e o conteúdo escolhido para reconhecimento, foram realizadas avaliações qualitativas e quantitativas, aspectos pedagógicos e metodológicos utilizando parâmetros criados por Vasconcelos e Souto (2003) em livros didáticos de ciência. Desta forma, inferimos que a partir das nossas avaliações os LD's presentes atualmente nas escolas, apesar de apresentar um bom arsenal de informações ainda se mostram ineficientes em algumas categorias, quando enquadrados em baixos níveis de avaliação, comprometendo o bom uso do mesmo. Levando em consideração a importância do LD para a os processos de ensino/aprendizagem em geral e por muitas vezes ser o único recurso didático presente na sala de aula, podemos considerar que ainda se a muito o que aperfeiçoar, pois, de fato os LD atuais não corresponde as necessidades da comunidade escolar a qual se destina.
\end{abstract}

Palavras-Chave: Educação; ensino de ciências; avaliação; livro didático; mamíferos.

\section{Introdução}

O livro didático pode ser definido, conforme Stray (1993), como um produto cultural composto, híbrido, que se encontra no "cruzamento da cultura, da pedagogia, da produção editorial e da sociedade”. Atualmente, os livros didáticos representam à principal, senão a única fonte de trabalho como material impresso na sala de aula, em muitas escolas da rede pública de ensino, tornando-se um recurso básico para o aluno e para o professor, no

\footnotetext{
${ }^{1}$ Licenciatura em Ciências Biológicas, Universidade Federal Rural de Pernambuco. Email: jdds.djalma@hotmail.com

2 Licenciatura em Ciências Biológicas, Universidade Federal Rural de Pernambuco.

3 Licenciatura em Ciências Biológicas, Universidade Federal Rural de Pernambuco.

${ }^{4}$ Licenciatura em Ciências Biológicas, Universidade Federal Rural de Pernambuco.

${ }^{5}$ Professor Doutor, Universidade Federal Rural de Pernambuco. Email: argusalmeida@gmail.com
} 
processo ensino-aprendizagem (LOPES, 2007).

O Programa Nacional do Livro Didático (PNLD) é uma iniciativa do Ministério da Educação (MEC) que tem por objetivo a aquisição e a distribuição universal e gratuita de livros didáticos para alunos das escolas públicas de ensino. Segundo Vasconcelos \& Souto (2003), grande parte da responsabilidade pela definição dos critérios de avaliação do livro didático ainda recaia sobre o PNLD, considerando que a comunidade científica deve participar na sugestão de novas abordagens, sobre a avaliação do material de apoio didático.

O livro didático (LD) é um instrumento importante para o ensino formal, mesmo não sendo o único material a ser utilizado em sala de aula, é decisivo para a qualidade do ensino. O professor em sua formação precisa de elementos que possibilitem reflexões a respeito das limitações do $\mathrm{LD}$, para reconhecer suas insuficiências e subsidiar novas práticas pedagógicas (LAJOLO, 1996).

Uma das condições primordiais para que o livro didático seja considerado bom é a de que ele esteja com os conceitos cientificamente corretos, com níveis de rigor e precisão apropriados à série que se destina; e possuir narrativas claras e compreensíveis, colocadas em uma linguagem interessante, que estimule o pensamento do educando. Em resumo, as narrativas devem sofrer uma transposição didática (MONTE, 2003).

Ainda de acordo com Lajolo (1996), por mais que o livro didático seja excelente, ele não é melhor que o professor que conhece a individualidade da classe e sabe qual conteúdo retrata melhor a realidade de seus alunos. Logo, o professor é o construtor de suas práticas em sala de aula, cabe a ele utilizar outros recursos ou estratégias pedagógicas de maneira a complementar ou superar tais insuficiências. Entretanto, é sabido que para que isso ocorra o professor deve ter em sua formação inicial elementos que propiciem uma leitura criteriosa do livro didático.

Atualmente, há uma crescente discussão a respeito da abordagem dos conteúdos dos livros didáticos. Apesar dos avanços, grande parte dos professores tem acesso limitado a recursos que possibilitem a análise dos livros (VASCONCELOS \& SOUTO, 2003). Pesquisas têm demonstrado que o livro didático de Biologia tem um papel determinante na organização curricular e na prática pedagógica dos professores (XAVIER et al, 2006). Os livros de Ciências têm uma função que os difere dos demais - a aplicação do método científico, estimulando a análise de fenômenos, o teste de hipóteses e a formulação de conclusões. Adicionalmente, deve propiciar ao aluno uma compreensão científica, filosófica e 
estética de sua realidade, oferecendo suporte no processo de formação dos indivíduos/cidadãos (VASCONCELLOS, 1993).

\section{Fundamentação Teórica}

Conforme os Parâmetros Curriculares Nacionais, no ensino de Ciências, os livros didáticos constituem um recurso de fundamental importância, pois muitas vezes, são o único material de apoio disponível para alunos e professores (BRASIL, 1996).

O Ensino de Zoologia, em geral usa formas simples de assimilação na construção do conhecimento: fotos, esquemas, modelos, desenhos, analogias e metáforas, que são de vital importância para promover a compreensão dos conteúdos no livro didático (SANTOS et al, 2010). Segundo Alves et al, 2006 o estudo de animais desperta um grande interesse por se tratar de um grupo diretamente relacionado ao cotidiano das pessoas e para tanto deve ser abordado de forma coerente e atualizada, para possibilitar a satisfatória assimilação por parte dos discentes com relação a determinados conteúdos.

O livro didático de Biologia tem sofrido críticas quanto ao tratamento unidirecional dos conteúdos, o dogmatismo e a apresentação dos conhecimentos como prontos e sem possibilidade de questionamento. Uma contradição quando se considera que os Parâmetros Curriculares Nacionais para o Ensino Médio sugerem que o ensino de Biologia deva estar voltado para o desenvolvimento de competências que permitam ao aluno lidar com as informações, sendo capaz de compreender o mundo e agir com autonomia, fazendo uso dos conhecimentos adquiridos (LOPES \& VASCONCELOS, 2012).

O conteúdo da zoologia nos livros didáticos inicialmente era introduzido nos livros de História Natural. A visão sobre os animais adotada nestas obras era predominantemente descritiva e classificatória, não havia aulas práticas ou atividades previstas, e o seu estudo forçava o estudante a decorar descrições e nomes científicos dos grupos. Estudos qualitativos estão ganhando notoriedade porque frequentemente são utilizados como único veículo de conteúdo, marcados pela forma fragmentária de organização (LOPES \& VASCONCELOS, 2012).

Por consequência de tudo isso já descrito, e outros pontos a serem apresentados, o presente trabalho tem como objetivo analisar horizontalmente livros didáticos do ensino de biologia usados em escolas do estado de Pernambuco, considerando seu conteúdo teórico, recursos visuais e adicionais e atividades propostas, utilizadas para o ensino da Classe Mammalia. 


\section{Metodologia}

\section{Critérios de escolha dos livros didáticos}

Para a análise e avaliação da abordagem da Classe Mammalia nos livros didáticos de biologia do ensino médio, foi usado como principal critério, edições usadas atualmente no ensino público e privado do estado de Pernambuco, em um total de seis títulos analisados (Ver tabela 1).

Tabela 1: Livros selecionados para a análise.

\begin{tabular}{|c|c|}
\hline LIVRO & REFERÊNCIA \\
\hline $\mathbf{A}$ & $\begin{array}{l}\text { Amabis, José Mariano, 1947. Biologia dos organismos / José Mariano } \\
\text { Amabis, Gilberto Rodrigues Martho. - 2. Ed. - São Paulo: Moderna, } \\
2004 .\end{array}$ \\
\hline $\mathbf{B}$ & $\begin{array}{l}\text { Lopes, Sônia. Bio: volume } 2 \text { / Sônia Lopes, Vivian Lavander Mendonça. } \\
\text { - 1. Ed. - São Paulo: Saraiva, } 2006 .\end{array}$ \\
\hline $\mathbf{C}$ & $\begin{array}{l}\text { Biologia: ensino médio, } 2^{\circ} \text { ano / organizadores Fernando Santiago dos } \\
\text { Santos, João Batista Vicentin Aguilar, Maria Martha Argel de Oliveira. - } \\
\text { 1. Ed. - São Paulo: Edições SM, 2010. (Coleção ser protagonista). }\end{array}$ \\
\hline D & $\begin{array}{l}\text { Ser protagonista: biologia, } 2^{\circ} \text { ano: ensino médio / obra coletiva concebida, } \\
\text { desenvolvida e produzida por Edições SM; editora responsável Tereza } \\
\text { Costa Osorio. - 2. Ed. - São Paulo: Edições SM, 2013. (Coleção ser } \\
\text { protagonista 2). }\end{array}$ \\
\hline $\mathbf{E}$ & $\begin{array}{l}\text { Silva Junior, César da. Biologia } 2 \text { / César da Silva Junior, Sezar Sasson, } \\
\text { Nelson Caldini Júnior. - 11. Ed. - São Paulo: Saraiva, } 2013 .\end{array}$ \\
\hline $\mathbf{F}$ & $\begin{array}{l}\text { Conexões com a Biologia. Editora responsável: Rita Helena } \\
\text { Bröckelmann; organizadora: Editora Moderna; obra coletiva concebida, } \\
\text { desenvolvida e produzida pela Editora Moderna. - 1. Ed, - São Paulo: } \\
\text { Moderna, 2013. }\end{array}$ \\
\hline
\end{tabular}

\section{Critérios para análise dos livros didáticos de biologia}

A partir dos livros selecionados e o conteúdo escolhido para análise, as avaliações qualitativas e quantitativas, aspectos pedagógicos e metodológicos foram feitas usando parâmetros utilizados por Vasconcelos e Souto (2003) em livros didáticos de ciência.

Para uma melhor verificação do foco norteador escolhido foram selecionados os seguintes eixos prioritários: 1 - Conteúdo Teórico; 2 - Recursos Visuais; 3 - Atividades 
Propostas e 4 - Recursos Adicionais (VASCONCELOS E SOUTO, 2003). Além disso, em consonância foram enquadrados dentro de cada eixo como fraco, regular, bom ou excelente.

Essa pesquisa foi conduzida pelos graduandos do curso de Licenciatura em Ciências Biológicas da Universidade Federal Rural de Pernambuco, auxiliados pelo professor Argus Vasconcelos de Almeida, ministrante da disciplina Prática de Biologia Animal.

\section{Resultados e Discussão}

\section{Conteúdo teórico}

Quanto à adequação a série dos LD's analisados B, C, D e E foram considerados bons, diferiram disso, o LD F considerado regular e o LD A excelente. A clareza do texto nos LD's B, C, D, E e F foi considerado boa, apenas o LD A foi considerado regular por apresentar bastante texto e poucos esclarecimento dos termos usando, considerando que o aluno já tenha um bom conhecimento prévio sobre o assunto.

Todos os LD’s apresentaram um nível de atualização satisfatório, sendo C, D, E e F considerado bons e A e B excelentes. No "Grau de coerência entra as informações apresentadas (ausência de contradições)" todos os LD's apresentaram excelência, apenas o LD F deferiu dos demais por apresentar texto complementar fora da classe que o capítulo destinava-se.

Dentro das outras observações encontradas podemos destacar que os LD's C e D dividem o capitulo com outra classe zoológica, além de apresentarem termos que divergem de todos os outros LD's analisados. Os LD's C e D são da mesma editora de edições diferentes, ambos apresentam os mesmos pontos negativos, apesar de trazer em sua segunda edição texto complementar diferente.

O LD E apresenta texto complementar interdisciplinar, enquanto o LD F o texto complementar fora da classe que o capítulo está destinado, como já mencionando anteriormente (Ver tabela 2).

Os critérios propostos visam identificar a adequação entre o conteúdo científico abordado nos livros e o universo cognitivo daqueles a quem se destinam, conforme Vasconcelos e Souto (2003). Por que, embora os livros didáticos passem por criteriosa revisão, ainda encontramos exemplos de contradições entre as informações apresentadas no 
conteúdo teórico. Detectar - e corrigir - tais contradições é função do professor de Ciências (VASCONCELOS E SOUTO, 2003).

TABELA 2: Critérios para análise do conteúdo teórico em livros didáticos de Ciências.

\begin{tabular}{|l|l|l|l|l|}
\hline \multicolumn{1}{|c|}{ PARÂMETRO } & \multicolumn{1}{|c|}{ FRACO } & \multicolumn{1}{|c|}{ REGULAR } & \multicolumn{1}{|c|}{ BOM } & EXCELENTE \\
\hline Adequação à série & & F & B, C, D e E & A \\
\hline $\begin{array}{l}\text { Clareza do texto (definições, } \\
\text { termos, etc.) }\end{array}$ & A & B, C, D, E e F & \\
\hline Nível de atualização do texto & & & C, D, E e F & A e B \\
\hline $\begin{array}{l}\text { Grau de coerência entra as } \\
\text { informações apresentadas } \\
\text { (ausência de contradições) }\end{array}$ & & & F , B, C, D e E & A \\
\hline Outros: Especificar & C, F, D e E & & \\
\hline $\begin{array}{l}\text { Apresenta textos } \\
\text { complementares? }\end{array}$ & A, B, C, D, E e F & & NãO \\
\hline
\end{tabular}

\section{Recursos visuais}

Nos parâmetros para "Qualidade das ilustrações", "Grau de relação com as informações contidas no texto" e "Inserção ao longo do texto (diagramação) " a maioria dos LD's receberam avaliação de bom a excelente. O LD B destacasse dentro disso, apresentando avaliação de regular por suas ilustrações serem de baixa qualidade. "Veracidade da informação contida na ilustração" todos os LD's recebem a classificação de excelente por aparecerem sempre com proposito, nunca de forma aleatória.

Foi surpreendente que em todos os LD's analisados apresentaram baixa avaliação dentro da possibilidade de contextualização. O "Grau de inovação (originalidade/criatividade)" dos LD's A, E e F foi considerado regular e de B, C, D bom. Apesar dos LD's C e D serem da mesma editora e só diferirem na edição apresentam diagramação diferente, em consequência disso, a segunda edição apresenta uma melhor apresentação do conteúdo visual. É importante destacar ainda que os LD’s C e D apresentam imagem de interação entre classes zoológicas. Nenhum dos LD's induzem a interpretação incorreta dos recursos visuais (Ver tabela 3).

De acordo com Vasconcelos e Souto (2013) a função das ilustrações é tornar as informações mais claras, estimulando a compreensão e a interação entre leitores e o texto 
científico. Desta forma os títulos que apresentam extremos - ilustrações em excesso ou escassas - podem resultar de deficiências metodológicas.

Tabela 3: Critérios para análise dos recursos visuais em livros didáticos de Ciências.

\begin{tabular}{|c|c|c|c|c|}
\hline PARÂMETRO & FRACO & REGULAR & BOM & EXCELENTE \\
\hline Qualidade das ilustrações & & B & $A, E$ e $F$ & C, D \\
\hline $\begin{array}{l}\text { Grau de relação com as } \\
\text { informações contidas no texto }\end{array}$ & & & B, E e F & $\mathrm{A}, \mathrm{C}, \mathrm{D}$ \\
\hline $\begin{array}{l}\text { Inserção ao longo do texto } \\
\text { (diagramação) }\end{array}$ & & B e F & $\mathbf{A}, \mathbf{C}, \mathbf{E}$ & D \\
\hline $\begin{array}{l}\text { Veracidade da informação } \\
\text { contida na ilustração }\end{array}$ & & & & $\begin{array}{l}\mathbf{A}, \mathbf{B}, \mathbf{C}, \mathbf{D}, \mathbf{E} \\
\text { e F }\end{array}$ \\
\hline $\begin{array}{l}\text { Possibilidade de } \\
\text { contextualização }\end{array}$ & & $\begin{array}{l}\mathrm{A}, \mathrm{B}, \mathrm{C}, \mathrm{D}, \mathrm{E} \\
\text { e F }\end{array}$ & & \\
\hline $\begin{array}{l}\text { Grau de inovação } \\
\text { (originalidade/criatividade) }\end{array}$ & & A, E e F & B, C, D & \\
\hline Outros: especificar & \multicolumn{4}{|l|}{$\mathbf{C}, \mathbf{D}, \mathbf{E}$} \\
\hline \multirow{2}{*}{$\begin{array}{l}\text { Induzem a interpretação } \\
\text { incorreta? }\end{array}$} & \multirow{2}{*}{\multicolumn{2}{|c|}{ SIM }} & \multicolumn{2}{|c|}{ NÃO } \\
\hline & & & \multicolumn{2}{|c|}{$\mathrm{A}, \mathrm{B}, \mathrm{C}, \mathrm{D}, \mathrm{E}$ e $\mathbf{F}$} \\
\hline
\end{tabular}

\section{Atividades propostas}

Para os parâmetros "Propõe atividades em grupo e/ou projetos para trabalho do tema exposto", "As atividades são facilmente executáveis" e "As atividades têm relação direta com o conteúdo trabalhado" todos os LD's cumpriam com esses requisitos, além de proporem questões ao final de cada capítulo.

Para o critério das questões terem enfoque multidisciplinar, priorizarem a problematização e proporem atividades em grupo e/ou projetos para trabalho do tema exposto os LD's C, D, E e F continham essas atividades que contemplam esses requisitos, ao mesmo tempo que os LD's A e B não pontuaram em nenhum desses quesitos.

Os LD's C, D, E e F indicaram fontes complementares de informação. Os LD's C e D continham indicação de filmes e sites, cumprindo outro requisito que é o estimulo da utilização de novas tecnologias.

O LD A propõe apenas questões de vestibular, o LD B contém um texto e atividade de outra classe zoológica dentro do capitulo de mamíferos. O LD F propõe leituras 
para pensamento crítico e atividades práticas (Ver tabela 4).

No ensino de Ciências, atividades práticas são fundamentais, afinal o desenvolvimento da capacidade investigativa e do pensamento científico são diretamente estimulados pela experimentação. Através de um experimento, o aluno tem oportunidade de formular e testar suas hipóteses, coletar dados, interpretá-los e elaborar suas próprias conclusões, baseadas na literatura sobre o tema. Uma experimentação permite ao aluno perceber que o conhecimento científico não se limita a laboratórios sofisticados, mas pode ser construído em sua sala de aula em parceria com professores e colegas. Ao se estimular a atividade experimental é necessário, evidentemente, observar sua pertinência pedagógica e a segurança daqueles diretamente envolvidos com sua execução (VASCONCELOS E SOUTO, 2003).

Tabela 4: Exemplos de atividades propostas utilizadas na complementação e contextualização do assunto discutido.

\begin{tabular}{|c|c|c|}
\hline ATIVIDADES & SIM & NÃO \\
\hline $\begin{array}{c}\text { Propõe questões ao final de cada } \\
\text { capítulo/tema? }\end{array}$ & $\mathbf{A}, \mathbf{B}, \mathbf{C}, \mathbf{D}, \mathbf{E}$ e $\mathbf{F}$ & \\
\hline $\begin{array}{l}\text { As questões têm enfoque } \\
\text { multidisciplinar? }\end{array}$ & $\mathrm{C}, \mathrm{D}, \mathrm{E}$ e F & A e B \\
\hline $\begin{array}{l}\text { As questões priorizam a } \\
\text { problematização? }\end{array}$ & C, D, E e F & A e B \\
\hline $\begin{array}{c}\text { Propõe atividades em grupo e/ou } \\
\text { projetos para trabalho do tema } \\
\text { exposto? }\end{array}$ & C, D, E e F & A e B \\
\hline $\begin{array}{l}\text { As atividades são isentas de risco } \\
\text { para alunos? }\end{array}$ & $\mathbf{A}, \mathbf{B}, \mathbf{C}, \mathrm{D}, \mathrm{E}$ e $\mathbf{F}$ & \\
\hline $\begin{array}{c}\text { As atividades são facilmente } \\
\text { executáveis? }\end{array}$ & $\mathbf{A}, \mathbf{B}, \mathbf{C}, \mathrm{D}, \mathrm{E}$ e $\mathbf{F}$ & \\
\hline $\begin{array}{l}\text { As atividades têm relação direta com } \\
\text { o conteúdo trabalhado? }\end{array}$ & $\mathrm{A}, \mathbf{B}, \mathrm{C}, \mathrm{D}, \mathrm{E}$ e $\mathbf{F}$ & \\
\hline $\begin{array}{c}\text { Indica fontes complementares de } \\
\text { informação? }\end{array}$ & C, D, E e F & A e B \\
\hline $\begin{array}{l}\text { Estimula a utilização de novas } \\
\text { tecnologias (ex. internet)? }\end{array}$ & C, D, E e F & A e B \\
\hline Outros: Especificar & \multicolumn{2}{|c|}{$A, B, E$ e $F$} \\
\hline
\end{tabular}




\section{Recursos adicionais}

A presença de recursos como "Cadernos de exercícios" e "Guia do professor" não tiveram como ser levantados. A presença de glossário foi unanime em todos os LD's pesquisados, já altas não continha em nenhum desses. "Guias de exercícios” estava presente em todos eles e se destacam podendo ser evidenciados através de atividades para responder no caderno, atividades, questões para discussão e vestibular.

Os LD's C, D e F contavam com propostas de experimentos práticos que mesmo sendo em sala de aula tinham cunho investigativo. Os LD's A, B e E não tinha nenhuma proposta de experimento (Ver tabela 5).

Definimos como recursos complementares ou adicionais os artifícios encontrados pelos autores para facilitar e direcionar a interação entre o livro e os professores e alunos. Glossários, atlas ilustrativos, cadernos de exercícios, guias de atividades experimentais, complementam as necessidades do aluno, oferecendo novas oportunidades de exercitar o conhecimento em construção e proporcionando melhor compreensão das informações trabalhadas ao longo da obra (VASCONCELOS E SOUTO, 2003).

Tabela 5: Exemplos de recursos complementares sugeridos em livros didáticos de Ciências.

\begin{tabular}{|c|c|c|}
\hline $\begin{array}{c}\text { RECURSOS } \\
\text { COMPLEMENTARES }\end{array}$ & SIM & NÃO \\
\hline Glossários & $\mathbf{A}, \mathbf{B}, \mathbf{C}, \mathbf{D}, \mathbf{E}$ e F & A, B, C, D, E e F \\
\hline Atlas & DADOS NÃO ENCONTRADOS \\
\hline Cadernos de exercícios & A, B, C, D, E e F & A, B e E \\
\hline Guias de exercícios & C, D e F & DADOS NÃO ENCONTRADOS \\
\hline Guias de experimentos & \multicolumn{2}{|c|}{. } \\
\hline Guia do professor & \multicolumn{2}{|c|}{} \\
\hline Outros: especificar &
\end{tabular}

\section{Conclusões}

Para finalizar a discussão e ser feito um melhor entendimento para os fatos encontrados, devemos nos prender ao que se faz necessário a função do LD na formação do aluno de ensino básico. Pois, a partir das atribuições que lhe é concedida ele precisa suprir as necessidades que venham a aparecer dentro da sala de aula, nos processos de 
ensino/aprendizagem, que envolve tanto a ação do professor sobre o livro, quanto do aluno sozinho ou sob a orientação do professor.

Os livros usados nessa pesquisa foram usados recentemente por escolas públicas e privadas do estado de Pernambuco, apesar de disponibilizarem de pouco recurso para a contextualização, serem de uma linguagem ainda rebuscada para a realidade da maioria dos alunos que hoje ocupam nossas escolas. A escolha dos LD's pelos professores apesar de ser um ponto positivo, ainda se mostra ineficiente quando evidenciado atrás de conceitos baixos a partir da nossa avaliação, somasse a isso o descompromisso das editoras em comtemplar todas as regiões com suas particularidades.

Por isso, podemos considerar que o estudo foi de grande importância para a avaliação de um grande instrumento de trabalho para os professores, por muitas vezes, único em algumas escolas. E além disso, serve para uma análise crítica reconhecendo os pontos positivos e negativos do produto verificado.

\section{Referências}

ALVES, L. F. A.; BUSARELLO, G. D.; GIANNOTTI, S. M. OS Artrópodes nos materiais didáticos utilizados em escolas da rede particular do ensino médio em cascavel, PR. Revista Varia Scientia, v. 06, n. 12, p. 107-120, 2006).

BRASIL. MEC/SEF. Guia de Livros Didáticos: 1ª a 4ª séries - PNLD 1996.

LAJOLO, M. Livro Didático: um (quase) manual de usuário. Brasília: Alberto, ano 16, n. 69, jan/mar. 1996.

LOPES, Alice Casimiro. Currículo e Epistemologia. Ijuí: Editora Unijuí, 2007, p. 205- 228.

LOPES, E. R.; VASCONCELOS, S. D.. Representação e distorções conceituais do conteúdo 'filogenia' em livros didáticos de biologia do ensino médio. Revista Ensaio, v.14, n.3, p.149-165, 2012.

MONTE, V.C. A Mata Atlântica nos livros didáticos de Ciências Naturais e Biologia. Recife, 2003. Dissertação (Mestrado) - Programa de Pós-Graduação em Ensino das Ciências, Universidade Federal Rural de Pernambuco, Recife, 2003.

SANTOS, S. C. S. et al. Analogias em livros didáticos de Biologia no ensino de Zoologia. Investigações em Ensino de Ciências, v. 15, n. 3, 2010.

STRAY, Chris. Quia Nominor Leo: Vers une sociologie historique du manuel. In: CHOPPIN, Alain (org.) Histoire de l'éducation. $\mathrm{n}^{\circ} 58$ (numéro spécial). Manuels scolaires, États et sociétés. XIXe-XXe siècles, Ed. INRP, 1993. 
VASCONCELOS, S. D.; SOUTO, E. O livro didático de ciências no ensino fundamental proposta de critérios para análise do conteúdo zoológico. Ciência \& Educação 9 (1): 93-104, 2003.

VASCONCELLOS, C. S. Construção do conhecimento em sala de aula. São Paulo: Libertad. 193 p. 1993.

XAVIER, M.C.F.; FREIRE, A.S.; MORAES, O.M. A Nova (Moderna) Biologia e a Genética nos livros didáticos de Biologia no Ensino Médio. Ciência e Educação, v.12, n.3, p.275-289, 2006. 